\title{
Ki-67 and Survivin as Predictive Factors for Rectal Cancer Treated with Preoperative Chemoradiotherapy
}

\author{
KOZO YOSHIKAWA ${ }^{1}$, MITSUO SHIMADA ${ }^{1}$, JUN HIGASHIJIMA ${ }^{1}$, TOSHIHIRO NAKAO ${ }^{1}$, MASAAKI NISHI ${ }^{1}$, \\ CHIE TAKASU $^{1}$, HIDEYA KASHIHARA ${ }^{1}$, SYOHEI ETO ${ }^{1}$ and YOSHIMI BANDO ${ }^{2}$ \\ Departments of ${ }^{1}$ Surgery, and ${ }^{2}$ Pathology, The University of Tokushima, Tokushima, Japan
}

\begin{abstract}
Aim: To evaluate the usefulness of Ki-67 index and survivin as predictive prognostic factors for rectal cancer treated with preoperative chemoradiotherapy. Patients and Methods: The Ki-67 index and survivin expression were examined in patients with stage II/III rectal cancer $(n=46)$ by immunohistochemistry. Patients were divided into a highgroup and a low-group for the Ki-67 index, and positive and negative groups for survivin expression. Overall and diseasefree survival were compared between the groups, and the correlation between Ki-67 index and survivin expression was assessed. Results: The 5-year disease-free survival rate of the group with high Ki-67 index was significantly lower than that of the group with low Ki-67 index (53\% and 88\%, $p=0.03)$, as was the 5-year overall survival rate $(68 \%$ and $100 \%, p=0.03)$. Findings for survivin were not significant. Conclusion: Ki-67 index and survivin may be useful biomarkers for rectal cancer with preoperative CRT.
\end{abstract}

Chemoradiotherapy (CRT) is being increasingly used in neoadjuvant therapy for rectal cancer. Preoperative CRT can preserve the anal sphincter, reduce the risk of local recurrence, and circumvent the need for invasive treatments, such as lateral lymph node dissection. Our group has used a novel protocol for neoadjuvant CRT combining S-1, which is a novel oral fluoropyrimidine, tegafur, 5-chloro-2,4- dihydroxypyridine (gimeracil; CDHP), and potassium oxonate (oteracil potassium). Tegafur is a prodrug for 5-FU (fluorouracil) that acts as an effector. CDHP reversibly inhibits the degradation of 5-FU by dihydropyrimidine dehydrogenase, resulting in prolonged high concentrations of 5-FU in the blood. S-1 has radiosensitizing

Correspondence to: Kozo Yoshikawa, MD, Ph.D., FACS, The Department of Surgery, The University of Tokushima, 3-18-15 Kuramoto-cho Tokushima, Japan. Tel: +81 886337139, Fax: +81 886319698, e-mail: yoshikawa.kozo@ tokushima-u.ac.jp

Key Words: Ki-67 index, survivin, pre-operative CRT, rectal cancer, survival. effects and enhances the effects of radiation. However, recent data have shown that preoperative CRT may result in a broad variety of tumor responses in rectal cancer. The success of radiation therapy is dependent on its ability to control tumor proliferation and induce cell death by causing irreparable cellular damage that ultimately triggers apoptosis. For patients with rectal cancer treated by neoadjuvant CRT, some reports have shown that a high number of apoptotic tumor cells in pretreatment biopsy specimens is a significant predictor for tumor response and long-term local control. The mechanisms behind the responses to CRT are not yet fully understood, although the ability of CRT to eradicate tumor cells appears to depend upon cell-cycle arrest, apoptosis, and DNA repair (1).

There are various markers that can be used to show the proliferative activity of cells. One of these markers is Ki-67, and the $\mathrm{Ki}-67$ index is the most commonly used indicator for measuring proliferation. Ki-67 is a nuclear non-histone protein that has been used to identify high-grade tumors in several cancer types. It is stably expressed in the $G_{1}, S, G_{2}$, and $\mathrm{M}$ phases, yet is absent in quiescent and resting cells $\left(\mathrm{G}_{0}\right)$. As such, Ki-67 expression has been reported to correlate with tumor cell proliferation rate (2). To date, many studies that have investigated the role of $\mathrm{Ki}-67$ as a prognostic marker in various cancer types have shown that Ki-67 positivity is an indicator of a higher risk of recurrence and a worse survival (1-4). To the best of our knowledge, there have been few reports of a relationship between Ki-67 and the prognosis of rectal cancer treated with CRT.

Survivin is a structurally unique member of the inhibitor of apoptosis protein family that regulates both cell-cycle progression and cell survival. It acts as a suppressor of apoptosis by inhibiting the activation of caspase-3/7, and it regulates the cell cycle at the $\mathrm{G}_{2} / \mathrm{M}$ phase. It is expressed in various human cancer types, such as lung, breast, prostate, ventricle, pancreas, and colon cancer, but it is not expressed in most normal adult human tissues; as such, it has attracted a great deal of interest as a potential drug target (5).

Although there are many markers that can be used to predict the response to preoperative CRT, few have been reported to 
be useful as predictive markers of prognosis. The aim of this study was to investigate the usefulness of the Ki-67 index and the expression of survivin as predictive prognostic factors for rectal cancer treated with preoperative CRT.

\section{Patients and Methods}

Patients. CRT is routinely offered to patients with locally advanced (T3 or node-positive) cancer at the time of diagnosis or to those who have very distal T2N0 cancer close to or involving the sphincter. All patients received CRT with a total dose of 4,000 cGy of pelvic irradiation, which was administered five times weekly, with a daily fraction of $200 \mathrm{cGy}$ utilizing a four-field technique. The top of the radiation field consisted of the bifurcation point of the aorta, and the bottom of the field was marked at least $4 \mathrm{~cm}$ below the tumor. The radiation field included the lateral nodes. Radiation was delivered concomitantly with S-1 (80-120 mg/day) on the same days. Surgical treatment was performed 6 to 8 weeks after the completion of preoperative CRT. Repeat examination was performed for post-neoadjuvant CRT evaluation. All patients completed the neoadjuvant treatment and underwent curative radical resection no earlier than 6 weeks after the completion of CRT.

Patients with stage II/III disease $(n=46)$ were examined in this study. All of the patients underwent surgical treatment at Tokushima University Hospital between 2003 and 2012, and all of them provided informed consent for participation in this study. The staging was principally based on the Japanese Classification of Colorectal Cancer.

Immunohistochemical staining for Ki-67. The resected tissue specimens were fixed in $10 \%$ formaldehyde, embedded in paraffin, and cut into 4 - $\mu$ m-thick sections that were deparaffinized with xylene, dehydrated with ethanol, and then treated with $0.03 \%$ hydrogen peroxide in methanol for $10 \mathrm{~min}$. For antigen retrieval, the sections were heated in $10 \mathrm{mM}$ citrate buffer $(\mathrm{pH} \mathrm{6})$ in a microwave. After cooling to room temperature, the sections were washed three times for 5 min each in phosphate-buffered saline, and then they were incubated with $1 \%$ goat serum to block nonspecific reactions. Next, the sections were incubated with primary antibodies against Ki-67 [anti-human Ki-67 antigen clone MIB-1 (M7240) diluted 1:100; Dako, Tokyo, Japan] for $60 \mathrm{~min}$ at room temperature. After washing three times for $5 \mathrm{~min}$ each in phosphate-buffered saline, the sections were subjected to a Dako REAL EnVision/HRP detection system (Dako) for $60 \mathrm{~min}$ at room temperature. The sections were then washed three times for $5 \mathrm{~min}$ each in phosphatebuffered saline, the peroxidase reaction was developed with 3,3'diaminobenzidine (Sigma-Aldrich, St Louis, MO, USA), and the sections were counterstained with $10 \%$ Mayer's hematoxylin. The sections were then dehydrated, treated with xylene, and coverslipped. A cut-off value of $30 \%$ was used.

Immunohistochemical staining for survivin. Slides were prepared as described above with the addition of $0.01 \%$ saponin in the permeabilization step. A 1:50 dilution of the primary antibody [polyclonal rabbit anti-survivin antibody (ab469); Abcam Cambridge, UK] was applied overnight in phosphate-buffered saline containing $5 \%$ bovine serum albumin. After two washes with a buffer, a 1:200 dilution of the secondary antibody (AlexaFluor 488 anti-rabbit antibody; Invitrogen, Karlsruhe, Germany) was added in a buffer with $5 \%$ albumin and the nuclei were stained with DAPI $(0.2 \mathrm{mg} / \mathrm{ml})$. Slides were covered with $25 \%$ glycerol in the buffer. The mean percentage of positively stained tumor cells was determined using an Image System (Nikon Digital Camera, DXM1200F; Nikon Tokyo, Japan) and assigned to one of the following categories: 0 ( $\leq 5 \%), 1(5 \%$ to $\leq 25 \%), 2$ ( $25 \%$ to $\leq 50 \%)$, $3(50 \%$ to $\leq 75 \%)$, or $4(>75 \%)$. The intensity of survivin immunostaining was scored as: 1 (weak), 2 (moderate), or 3 (intense). The score for the percentage of positively stained tumor cells and the score for the staining intensity for cytoplasm and nucleus were then added to obtain the total score for each case, ranging from 0 to 10 . The immunostaining results were evaluated by two independent pathologists in a blinded fashion without any knowledge of the clinicopathological or biological information of the cases. Because of the limited number of patients in this study and to facilitate further statistical analyses, the weighted survivin scores were arbitrarily dichotomized: negative-survivin expression was classified as a survivin score of 4 or below, and positivesurvivin expression was classified as a score of 5 or above.

Statistical analyses. Fisher's exact test and the Wilcoxon test were performed as appropriate. Continuous variables are expressed as the means \pm standard deviation (SD). JMP 8 software (SAS Institute, Cary, NC, USA) was used for all statistical analyses. Survival endpoints measured from the operation for cancer. Continuous variables were compared by Mann-Whitney $U$-tests, and categorical data were compared by chi-square tests. Survival curves were calculated by the Kaplan-Meier method and compared by log-rank tests. The prognostic potentials of the parameters were analyzed by univariate analysis. Relative risk and $95 \%$ confidence intervals were estimated with the Cox proportional hazards model with stepwise forward selection. Statistical significance was defined as $p<0.05$.

\section{Results}

Immunohistochemistry. Examples of the immunohistochemical analysis of Ki-67 and survivin expression are shown in Figure 1.

Immunohistochemical analysis of Ki-67 expression. There were 17 patients in the group with low Ki-67 index and 29 patients in the group with high Ki-67 index. There was no significant difference between the two groups in clinicopathological factors (Table I).

Survival curves according to $\mathrm{Ki}$-67 expression. Only high $\mathrm{Ki}$ 67 expression was found to be a prognostic factor for overall survival. The group with high Ki-67 index had a significantly poorer prognosis than the group with low Ki-67 index; the 5year survival rate of the group with low Ki-67 index was $100 \%$, while that of the group with high Ki-67 index was $68 \%$ $(p=0.03)$ (Figure 2A; Table II). For disease-free survival, univariate analysis revealed that the tumor size and Ki-67 expression were prognostic factors, while multivariate analysis revealed that $\mathrm{Ki}-67$ expression was an independent prognostic factor (hazard ratio $=2.46,95 \%$ confidence interval $=1.08-5.87$, $p=0.03)$. The group with high Ki-67 index had a significantly poorer prognosis than that with low Ki-67 index; the 5-year 

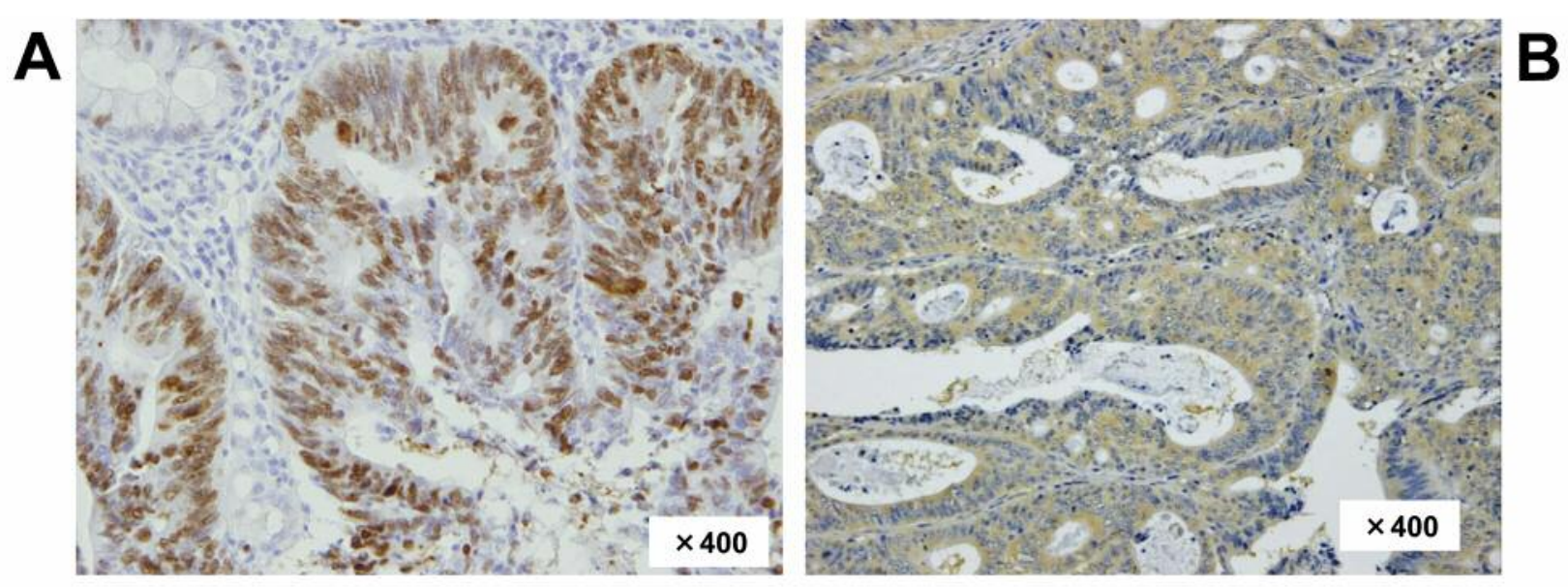

Figure 1. Examples of samples with positive immunohistochemistry results. A: Ki-67. B: Survivin (score 7).

disease-free survival rate of the group with low Ki-67 index was $88 \%$, while that of the group with high Ki-67 index was $53 \%(p=0.03)$ (Figure 2B; Table III).

Correlation between $\mathrm{Ki}-67$ and survivin. There were 23 patients with survivin-positive disease and 23 with survivin-negative. There was no significant difference in disease-free or overall survival between the two groups. A significant correlation was found between high Ki-67 expression and survivin: $78 \%$ of the patients with survivin-positive disease had high Ki-67 expression, while only $47 \%$ of those with survivin-negative disease had high Ki-67 expression ( $p=0.03$ ) (Figure 3).

\section{Discussion}

The results showed that a high Ki-67 index may be used to predict a poor prognosis for patients with rectal cancer undergoing CRT therapy, and that the expression of Ki-67 is correlated with survivin.

Preoperative CRT was introduced to improve the prognosis of surgery. The use of preoperative CRT for locally advanced rectal cancer has been strongly supported by the results of the German Rectal Cancer Trial, which demonstrated that preoperative CRT reduced the occurrence of local recurrence, lowered the rates of short-term and chronic toxicities, and improved the rate of sphincter preservation (3). Additionally, preoperative CRT for advanced low rectal cancer has been shown to increase the probability of tumor resectability, reduce the risk of local recurrence, and improve both disease-free and overall survival. Ki-67 antigen is expressed during all of the active phases of the cell cycle $\left(\mathrm{G}_{1}, \mathrm{~S}, \mathrm{G}_{2}\right.$, and mitosis) and has become a widely used marker for determining the proliferative potential of tumors. Rapidly proliferating cells typically respond more dramatically to radiation-induced cell damage
Table I. Clinicopathological factors of patients included in this study by Ki-67 expression.

\begin{tabular}{|c|c|c|c|}
\hline \multirow[b]{2}{*}{ Factor } & \multicolumn{2}{|c|}{$\mathrm{Ki}-67, \mathrm{n}$} & \multirow[b]{2}{*}{$p$-Value } \\
\hline & Low $(n=17)$ & High $(n=29)$ & \\
\hline Age: $<65 />65$ years & $7 / 10$ & $14 / 15$ & 0.40 \\
\hline Gender: $\mathrm{M} / \mathrm{F}$ & $11 / 6$ & $21 / 8$ & 0.58 \\
\hline Tumor size: $<2 \mathrm{~cm} />2 \mathrm{~cm}$ & $8 / 9$ & $7 / 22$ & 0.10 \\
\hline Stage*: II/III & $10 / 7$ & $16 / 13$ & 0.80 \\
\hline Grade*: $0,1 / 2,3$ & $8 / 9$ & $21 / 8$ & 0.08 \\
\hline Tumor differentiation: Well/other & $7 / 10$ & $10 / 19$ & 0.81 \\
\hline ly: $0 / 1,2$ & $13 / 4$ & $18 / 11$ & 0.30 \\
\hline v: $0 / 1,2$ & $8 / 9$ & $12 / 17$ & 0.70 \\
\hline
\end{tabular}

M: Male; F: female; ly: lymphatic invasion v: venous invasion. *Principally based on the Japanese Classification of Colorectal Cancer.

(4). Ki-67 has been used to assess cell proliferation and activity, and a correlation between Ki-67 and chemosensitivity has been reported (5-8). However, to the best of our knowledge, this is the first report on the expression levels of Ki-67 in rectal cancer treated with CRT; we found that a high expression of $\mathrm{Ki}-67$ is correlated with a poorer prognosis in patients with rectal cancer treated with CRT.

In addition, we examined the expression of survivin as it regulates both cell-cycle progression and cell survival. We found a positive correlation between Ki-67 and survivin. This significant direct correlation between Ki-67 and survivin suggests that the latter may have a pro-mitotic function. As a member of the inhibitor of apoptosis protein family, survivin may inhibit apoptosis in two ways: direct inhibition of caspase-3/7 activity, and negative regulation of cell apoptosis induced by various stimulation processes. Survivin with 

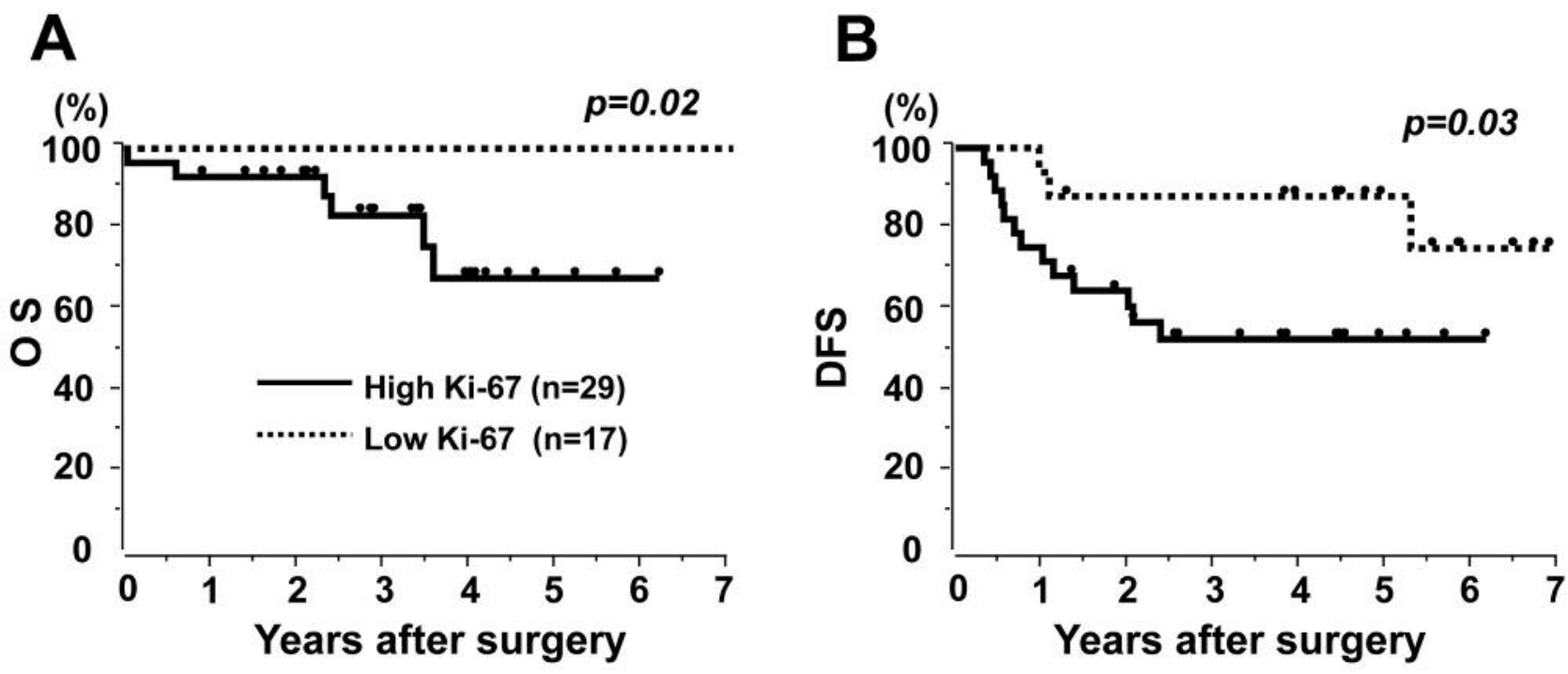

Figure 2. Survival curves. A: Overall survival (OS). B: Disease-free survival (DFS).

Table II. Overall survival (OS) of patients included in this study.

\begin{tabular}{lcc}
\hline Factor & 5 Year OS $(\%)$ & $p$-Value \\
\hline Age: $<65 />65$ years & $84.4 / 83.0$ & 0.88 \\
Gender: M/F & $83.1 / 84.4$ & 0.74 \\
Tumor size: $<2 \mathrm{~cm} />2 \mathrm{~cm}$ & $83.0 / 82.3$ & 0.62 \\
Stage*: II/III & $86.0 / 78.3$ & 0.61 \\
Grade*: 0,1/2,3 & $80.7 / 85.5$ & 0.83 \\
Tumor differentiation: Well/other & $82.2 / 80.7$ & 0.77 \\
ly: 0/1,2 & $87.2 / 72.7$ & 0.23 \\
v: 0/1,2 & $78.0 / 90.3$ & 0.46 \\
Ki-67: Low/high & $100 / 68.0$ & 0.03 \\
\hline
\end{tabular}

M: Male; F: female; ly: lymphatic invasion v: venous invasion. *Principally based on the Japanese Classification of Colorectal Cancer.

cyclin-dependent kinase CDK4/2 can block the apoptosis signaling transduction pathway. It can act directly on caspases by inhibiting caspase-3/7 activity, and also indirectly by inhibiting caspases through P21 interactions (9-11). Studies have shown that survivin along with cell-cycle regulatory factors can induce CDK/cyclin-E activation and ribosomal phosphorylation through CDK4 interactions. Ribosomal phosphorylation rapidly induces cells to enter the cell cycle and accelerates the progression of the $G_{1} / S$ phase, causing P21 release from the surviving-CDK4 complex combined with mitochondrial pro-caspase- 3 to inhibit caspase- 3 activity and prevent mitochondrial release of cytochrome $c$, thereby inhibiting apoptosis (12). Studies have also shown that survivin can inhibit the BCL2-associated X, apoptosis regulator (BAX)- and FAS-mediated apoptosis pathways. In
Table III. Disease-free survival (DFS) of patients included in this study.

\begin{tabular}{lcc}
\hline Factor & 5-Year DFS $(\%)$ & $p$-Value \\
\hline Age: $<65 />65$ years & $62.4 / 65.0$ & 0.66 \\
Gender: M/F & $67.1 / 64.4$ & 0.65 \\
Tumor size: $<2 / \geq 2 \mathrm{~cm}$ & $86.0 / 56.3$ & 0.03 \\
Stage*: II/III & $72.0 / 59.3$ & 0.32 \\
Grade*: 0,1/2,3 & $61.7 / 74.5$ & 0.19 \\
Tumor differentiation: Well/other & $65.2 / 63.7$ & 0.77 \\
ly: 0/1,2 & $68.2 / 63.4$ & 0.68 \\
v: 0/1,2 & $60.2 / 71.7$ & 0.27 \\
Ki-67: Low/high & $88.0 / 53.2$ & 0.03 \\
\hline
\end{tabular}

M: Male; F: female; ly: lymphatic invasion v: venous invasion. *Principally based on the Japanese Classification of Colorectal Cancer.

addition, Ki-67 and survivin form interaction networks, and survivin and p53 are linked through cell-cycle regulatory signaling pathways $(12,13)$.

There are currently many markers that predict the response to preoperative CRT, e.g., p21, survivin, Ki-67, BAX, and thymidylate synthase (13). However, there has been a few reports of predictive marker of prognosis. In this study, although survivin was not found to be a predictive factor of prognosis, Ki-67 was; this represents a novel finding. Predictive factors that can be assessed in surgically resected specimens are expected to be very useful in identifying individuals with a poor prognosis. Although a clear cut-off value for Ki-67 in rectal cancer treated with CRT has not yet been established, this study successfully distinguished the patients with a better prognosis from those 


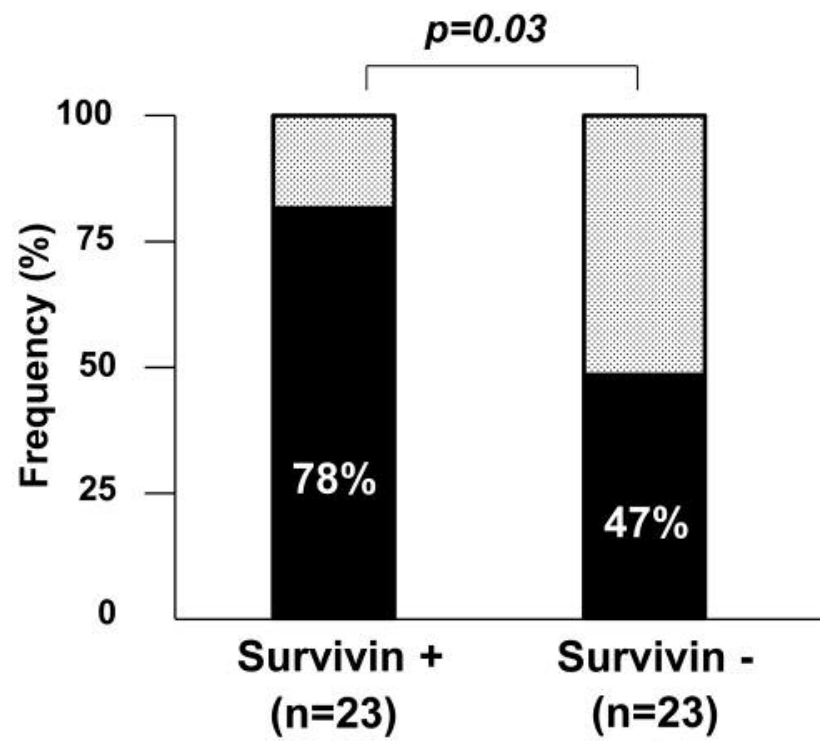

High Ki-67

Low Ki-67

Figure 3. Correlation between Ki-67 and survivin.

with a worse prognosis after CRT using a cut-off value of $30 \%$. A large prospective study is required to re-evaluate the validity of Ki-67 as a prognostic factor and its cut-off value.

Limitations of this study include its retrospective design and the fact that the specimens were obtained after CRT. CRT may influence the cell cycle of rectal cancer cells, so a pre-CRT specimen should also be examined, but the amount of the tissue was limited in this study because the biopsy material obtained was small.

\section{Conclusion}

A high Ki-67 index and the expression of survivin may be useful prognostic biomarkers for rectal cancer treated with preoperative CRT.

\section{Conflicts of Interest}

Drs. Kozo Yoshikawa, Mitsuo Shimada, Jun Higashijima, Toshihiro Nakao, Masaaki Nishi, Chie Takasu, Syohei Eto, Hideya Kashihara have no conflicts of interest or financial ties to disclose.

\section{References}

1 Hayashi H, Beppu T, Sakamoto Y, Miyamoto Y, Yokoyama N, Higashi T, Nitta H, Hashimoto D, Chikamoto A and Baba H: Prognostic value of Ki-67 expression in conversion therapy for colorectal liver-limited metastases. Am J Cancer Res 15: 12251233, 2015.
2 Sueta A, Yamamoto Y, Hayashi M, Yamamoto S, Inao T, Ibusuki $\mathrm{M}$, Murakami $\mathrm{K}$ and Iwase $\mathrm{H}$ : Clinical significance of pretherapeutic Ki67 as a predictive parameter for response to neoadjuvant chemotherapy in breast cancer: is it equally useful across tumor subtypes? Surgery 155: 927-935, 2014.

3 Takasu C, Shimada M, Kurita N Iwata T, Sato H, Nishioka M, Morimoto $\mathrm{S}$, Yoshikawa $\mathrm{K}$, Miyatani $\mathrm{T}$, Kashihara $\mathrm{H}$, Utsunomiya $\mathrm{T}$ and Uehara $\mathrm{H}$ : Survivin expression can predict the effect of chemoradiotherapy for advanced lower rectal cancer. Int J Clin Oncol 18: 869-876, 2013.

4 Okumura Y, Nishimura R, Nakatsukasa K, Yoshida A, Masuda N, Tanabe M, Shien T, Tanaka S, Arima N, Komoike Y, Taguchi $\mathrm{T}$, Iwase $\mathrm{T}$, Inaji $\mathrm{H}$ and Ishitobi M: Change in estrogen receptor, HER2, and Ki-67 status between primary breast cancer and ipsilateral breast cancer tumor recurrence. Eur J Surg Oncol 41: 548-552, 2015.

5 Kucukgoz Gulec U, Gumurdulu D, Guzel AB, Paydas S, Seydaoglu G, Acikalin A, Khatib G, Zeren H, Vardar MA and Altintas A: Prognostic importance of survivin, Ki-67, and topoisomerase II $\alpha$ in ovarian carcinoma. Arch Gynecol Obstet 289: 393-398, 2014.

6 Nishimura R, Osako T, Nishiyama Y, Tashima R, Nakano M, Fujisue M, Toyozumi Y and Arima N: Prognostic significance of $\mathrm{Ki}-67$ index value at the primary breast tumor in recurrent breast cancer. Mol Clin Oncol 2: 1062-1068, 2014.

7 Brun SN, Markant SL, Esparza LA, Garcia G, Terry D, Huang JM, Pavlyukov MS, Li XN, Grant GA, Crawford JR, Levy ML, Conway EM, Smith LH, Nakano I, Berezov A, Greene MI, Wang Q and Wechsler-Reya RJ: Survivin as a therapeutic target in Sonic hedgehog-driven medulloblastoma. Oncogene 34: 3770-3779, 2015.

8 Lin Q, Liu Y, Chen H, Liu Y, Tang Q, Liu J and Chen H: Survivin, Ki-67 and tumor grade as predictors of response to docetaxel-based neoadjuvant chemotherapy in locally advanced breast cancer. Mol Clin Oncol 1: 839-844, 2013.

9 Hur H, Kim NK, Min BS, Baik SH, Lee KY, Koom WS, Ahn JB and Kim H: Can a biomarker-based scoring system predict pathologic complete response after preoperative chemoradiotherapy for rectal cancer? Dis Colon Rectum 57: 592-601, 2014.

$10 \mathrm{Lim} \mathrm{SB}$, Yu CS, Hong YS, Kim TW, Kim JH and Kim JC: Long-term outcomes in patients with locally advanced rectal cancer treated with preoperative chemoradiation followed by curative surgical resection. J Surg Oncol 106: 659-666, 2012.

11 Nakamura T, Yamashita K, Sato T, Ema A, Naito M and Watanabe M: Neoadjuvant chemoradiation therapy using concurrent S-1 and irinotecan in rectal cancer: impact on longterm clinical outcomes and prognostic factors. Int J Radiat Oncol Biol Phys 89: 547-555, 2014.

12 Fu DR, Kato D, Watabe A, Endo Y and Kadosawa T: Prognostic utility of apoptosis index, Ki-67 and survivin expression in dogs with nasal carcinoma treated with orthovoltage radiation therapy. J Vet Med Sci 76: 1505-1512, 2014.

13 He WL, Li YH, Yang DJ, Song W, Chen XL, Liu FK, Wang Z, Li W, Chen W, Chen CY, He YL and Zhan WH: Combined evaluation of centromere protein $\mathrm{H}$ and $\mathrm{Ki}-67$ as prognostic biomarker for patients with gastric carcinoma. Eur J Surg Oncol 39: 141-149, 2013.

Received December 12, 2017

Revised January 19, 2018 Accepted January 24, 2018 